\title{
APLICAÇÕES DA CURVA DE RESISTÊNCIA NO CONTROLE DA QUALIDADE FÍSICA DE UM SOLO SOB PASTAGEM ${ }^{1}$
}

\author{
SILVIA IMHOFF ${ }^{2}$, ALVARO PIRES DA SILVA $^{3}$ e CÁSSIO ANTONIO TORMENA ${ }^{4}$
}

\begin{abstract}
RESUMO - A degradação da qualidade física do solo pode estar associada com a compactação causada pelo pisoteio dos animais. A resistência do solo à penetração (RP) é um parâmetro físico utilizado para estabelecer o grau de compactação do solo. Contudo, esta varia com a umidade $(\theta)$ e densidade do solo (Ds). O objetivo deste trabalho foi obter a curva de resistência do solo e utilizá-la na avaliação da qualidade física do solo num sistema de pastejo intensivo rotacionado de capim-elefante. A curva de resistência do solo foi determinada por meio de 48 amostras indeformadas, obtidas na profundidade de 0-10 cm numa Terra Roxa Estruturada utilizada com pastagem em sistema intensivo de exploração. Os resultados demonstraram correlação negativa entre a RP e $\theta$, e correlação positiva entre RP e Ds. Estimativas indicaram que no potencial de $-0,01 \mathrm{MPa}$ a RP não atinge valores considerados restritivos ao crescimento radicular. Entretanto, no potencial de $-0,3 \mathrm{MPa}$, a RP atinge níveis limitantes em toda a área. Quanto ao sistema de manejo e a espécie estudada, os resultados sugerem que a curva de resistência do solo pode ser utilizada para orientar as práticas de manejo visando à manutenção de uma qualidade física do solo adequada para o crescimento das plantas.
\end{abstract}

Termos para indexação: compactação do solo, densidade do solo, umidade do solo, resistência mecânica do solo, gramíneas forrageiras, crescimento de raízes.

\section{APPLICATIONS OF THE RESISTANCE CURVE \\ IN THE CONTROL OF THE PHYSICAL QUALITY OF SOILS UNDER GRASS}

\begin{abstract}
The degradation of the physical quality of soils has been associated with the soil compaction caused by animal trampling. The soil resistance to penetration (SRP) is a soil physical parameter used to assess the soil compaction degree. However, SRP varies with soil moisture $(\theta)$ and bulk density $\left(\mathrm{D}_{\mathrm{b}}\right)$. The objective of this research was to quantify the soil resistance curve and use it to evaluate the physical quality of a soil under short duration grazing system of elephant grass. The soil resistance curve was measured using forty-eight undisturbed cores taken at the $0-10 \mathrm{~cm}$ depth of a Kandiudalfic Eutrudox used with elephant grass in intensive system of exploration. The results indicated a negative correlation among SRP and $\theta$ as well as a positive correlation between SRP and $\mathrm{D}_{\mathrm{b}}$. Predictions indicated that at soil matric potential equal to $-0.01 \mathrm{MPa}$ the SRP did not reach critical values to root growth. Nevertheless, at the soil matric potential equal to $-0.3 \mathrm{MPa}$, the SRP reached limiting values in the whole area. For the management system and the studied species, the results suggest that soil resistance curve may be used to guide soil management practices for maintaining a good soil physical quality for grass growth.

Index terms: soil compaction, soil density, soil water content, soil strength, feed grasses, root growth.
\end{abstract}

\footnotetext{
${ }^{1}$ Aceito para publicação em 5 de agosto de 1999 .

Extraído da dissertação de mestrado do primeiro autor apresentada à Escola Superior de Agricultura "Luiz de Queiroz" (ESALQ), da Universidade de São Paulo (USP).

${ }^{2}$ Eng. Agrôn., Dep. de Solos e Nutrição de Plantas, ESALQ/ USP, Av. Pádua Dias, 11, Caixa Postal 9, CEP 13418-900 Piracicaba, SP. E-mail: scigiave@carpa.ciagri.usp.br

${ }^{3}$ Eng. Agrôn., Ph.D., Dep. de Solos e Nutrição de Plantas, ESALQ/USP. Bolsista do CNPq.

E-mail: apisilva@carpa.ciagri.usp.br

${ }^{4}$ Eng. Agrôn., Dep. de Agronomia, Universidade Estadual de Maringá (UEM), CEP 87020-900 Maringá, PR E-mail:catormen@uem.br
}

\section{INTRODUÇÃO}

O potencial produtivo das pastagens é determinado por fatores relacionados ao clima, ao solo e à espécie envolvida. O solo pode impor limitações ao crescimento das plantas pela capacidade de suprilas com nutrientes, água e oxigênio. Limitações à produtividade das pastagens podem estar relacionadas ao grau de fertilidade do solo e à ocorrência de $\mathrm{Al} \mathrm{e}$ Mn em níveis tóxicos (Haag \& Dechen, 1986). No entanto, atributos físicos do solo favoráveis ao cres- 
cimento do sistema radicular são necessários para a obtenção e manutenção de elevadas produtividades. Os solos devem possuir suficiente espaço poroso para o movimento de água e gases e resistência favorável à penetração das raízes. Neste contexto, a compactação causada pelo pisoteio dos animais concorre para a redução da produtividade e longevidade das pastagens.

A compactação do solo é caracterizada pela redução de volume do solo quando uma pressão externa é aplicada (Horn \& Lebert, 1994; Hakansson \& Voorhees, 1998). Em pastagens, a compactação pode estar associada às altas pressões exercidas pelo pisoteio de animais. Willatt \& Pullar (1983) assinalaram que bois da raça Jersey podem exercer pressões de 0,2 MPa, enquanto que as pressões aplicadas no solo por ovinos e caprinos foram de $0,08 \mathrm{MPa}$ e $0,06 \mathrm{MPa}$, respectivamente. Na literatura são mencionados valores que variam entre $0,25 \mathrm{MPa}$ e $0,49 \mathrm{MPa}$ para bovinos de $400 \mathrm{~kg}$ a $500 \mathrm{~kg}$, podendo atingir a profundidade de $5 \mathrm{~cm}$ a $10 \mathrm{~cm}$ (Carvalho, 1976; Proffitt et al., 1993). A compactação induz aumento de densidade do solo (Carvalho, 1976; Chanasyk \& Naeth, 1995; Azenegashe et al., 1997), diminuição da porosidade total, e alteração na distribuição dos tamanhos dos poros (Willatt \& Pullar, 1983; Dexter, 1988) e das propriedades hidráulicas do solo (Horton et al., 1994). Outros efeitos têm sido observados, como modificações na estabilidade e tamanho dos agregados (Warren et al., 1986) e aumento na resistência do solo à penetração das raízes (Murphy et al., 1995). A extensão e natureza das modificações nos atributos do solo ocasionadas pelo pisoteio dependem da intensidade de pisoteio, da umidade e tipo de solo. O solo muito úmido está sujeito a uma maior degradação física (Haveren, 1983; Proffit et al., 1993).

Independentemente da distribuição espacial e do perfil, a compactação cria um ambiente desfavorável para o crescimento das plantas. Vários estudos ressaltam a importância de se avaliar os atributos físicos do solo, uma vez que as raízes das plantas que crescem sob condições de estresse parecem ser capazes de identificar essas condições e de enviar sinais para a parte aérea, as que controlam a expansão foliar (Davies \& Zhang, 1991; Tardieu, 1994). Em solos compactados e sob secamento, esses sinais de inibição ocorrem em associação com o aumento da resistência do solo à penetração das raízes (Passioura \& Gardner, 1990). Valores de resistência mecânica do solo entre $2 \mathrm{MPa}$ e $3 \mathrm{MPa}$ são considerados limitantes ao desenvolvimento radicular para várias culturas anuais, como trigo, milho e algodão (Taylor \& Burnett, 1964; Gupta \& Allmaras, 1987; Vepraskas, 1994). Quanto à determinação da resistência à penetração, Benghough \& Mullins (1990) assinalaram que os penetrômetros fornecem boas estimativas da resistência mecânica do solo à penetração das raízes. Apesar das vantagens do uso dos penetrômetros, a resistência varia diretamente com a densidade do solo e inversamente com o conteúdo de água do solo (Busscher et al., 1997), dificultando a interpretação caso esses fatores não sejam levados em consideração (Cassel et al., 1978).

Um modelo para avaliar a resistência do solo à penetração das raízes como uma função não linear da umidade e densidade do solo foi proposto por Busscher (1990) e utilizado por Silva et al. (1994), obtendo excelentes resultados. A relação da resistência mecânica do solo com a umidade e densidade do solo, descrita como curva de resistência do solo, pode ser utilizada para fazer inferências sobre as condições estruturais do solo em relação as resistências críticas para o crescimento das plantas. Deste ponto de vista, a avaliação da resistência do solo e a determinação da curva de resistência é importante no estudo do efeito da compactação sobre as condições físicas do solo, podendo ser utilizadas para orientar o manejo e o controle da qualidade física do solo.

O objetivo deste trabalho foi obter a curva de resistência do solo e utilizá-la na avaliação da qualidade física do solo num sistema de pastejo intensivo de capim-elefante.

\section{MATERIAL E MÉTODOS}

O trabalho foi desenvolvido na área experimental do Departamento de Zootecnia da Escola Superior de Agricultura "Luiz de Queiroz", no município de Piracicaba, Estado de São Paulo, localizado entre as coordenadas geográficas $22^{\circ} 42^{\prime}$ de latitude sul e $47^{\circ} 38^{\prime}$ de longitude oeste, e a $538 \mathrm{~m}$ de altitude. O clima, segundo a classificação de Köppen, corresponde a Cwa (mesotérmico úmido subtropical de inverno seco). A precipitação pluvial média 
anual é de $1.253 \mathrm{~mm}$. A temperatura média anual é de $21,1^{\circ} \mathrm{C}$, com média do mês mais frio de $10^{\circ} \mathrm{C}$, e a do mês mais quente, de $30^{\circ} \mathrm{C}$ (Ometto, 1989). O solo na área experimental está classificado como Terra Roxa Estruturada eutrófica $\left(\mathrm{TRE}_{\mathrm{e}}\right.$ ), e apresenta $27 \%$ de areia, $55 \%$ de argila e $18 \%$ de silte, e $49,70 \mathrm{~g} \mathrm{~kg}^{-1}$ de matéria orgânica.

A área possui sistema intensivo de exploração da pastagem com rotação dos animais em piquetes de $1.000 \mathrm{~m}^{2}$ (20 m por $50 \mathrm{~m}$ ). A pastagem foi estabelecida em 1963/64, utilizando o capim-elefante (Pennisetum purpureum Schum) como forrageira. O pastejo rotacionado vem sendo utilizado desde $1971 \mathrm{com}$ animais de $450 \mathrm{~kg}$ a $600 \mathrm{~kg}$. A taxa de lotação empregada tem sido incrementada com os anos, de acordo com o aumento de produtividade do capim-elefante, que é, atualmente, de 11 a 12 unidades animais por ha (UA ha ${ }^{-1}$ ) durante o verão e 2,5 a 3 UA ha $^{-1}$ durante o inverno. O ciclo de pastejo é de 45 dias, com os animais permanecendo nos piquetes durante um dia.

Para a realização do estudo foi escolhido um piquete situado na posição topográfica da meia encosta. $\mathrm{Na}$ área foram localizados 24 pontos de amostragens, distribuídos regularmente com espaçamento de $4 \mathrm{~m}$ por $8 \mathrm{~m}$, resultando numa malha de quatro colunas e seus linhas. Em cada ponto foram coletadas duas amostras: uma, na proximidade das touceiras de capim-elefante (área com maior concentração de raízes), e a outra, a uma distância de cerca de $50 \mathrm{~cm}$ (área com solo descoberto entre duas touceiras), na camada superficial de 0 a $10 \mathrm{~cm}$. Esse sistema foi adotado com o objetivo de incorporar na amostragem a variabilidade da estrutura do solo, induzida pelas raízes e pelo pisoteio dos animais. As bordaduras foram de $4 \mathrm{~m} \mathrm{e} \mathrm{4,5} \mathrm{m.} \mathrm{Em} \mathrm{cada}$ ponto foram coletadas amostras indeformadas, em anéis volumétricos de $7 \mathrm{~cm}$ de altura por $7 \mathrm{~cm}$ de diâmetro, totalizando 48 amostras.

As amostras foram saturadas com água por 24 horas e em seguida submetidas a diferentes potenciais mátricos $(\psi)$ em mesa de tensão e em panelas de pressão de Richards conforme Klute (1986), sendo utilizadas seis amostras por potencial. Foram aplicados os $\psi$ de $-0,001,-0,003,-0,005$, $-0,01,-0,03,-0,07,-0,1$ e $-1,5 \mathrm{MPa}$ com o objetivo de simular um gradiente de umidade entre as amostras. Após atingir o equilíbrio de cada amostra, foi medida a resistência do solo à penetração, por meio de um penetrômetro eletrônico, com velocidade constante de penetração de $1 \mathrm{~cm} \mathrm{~min}{ }^{-1}$, e um cone com diâmetro de base de $4 \mathrm{~mm}$ e semi-ângulo de $30^{\circ}$. Esse penetrômetro é equipado com um atuador linear e célula de carga de $20 \mathrm{~kg}$ acoplada a um microcomputador para a aquisição dos dados (Tormena et al., 1998). No centro geométrico de cada amostra, uma leitura de resistência foi obtida a cada $0,01 \mathrm{~cm}$, na profundidade de 0,5 a $6,5 \mathrm{~cm}$, perfazendo um total de 600 leitu- ras. Foi calculada a média da resistência do solo à penetração de cada amostra. Após a determinação da resistência à penetração, as amostras foram secadas em estufa a $105^{\circ} \mathrm{C}$ por 24 horas e determinada a densidade do solo de acordo com Blake \& Hartge (1986).

Os dados de resistência à penetração foram ajustados em relação a umidade volumétrica $(\theta)$ e a densidade do solo (Ds) utilizando o modelo não linear proposto por Busscher (1990):

$\mathrm{RP}=\mathrm{a} \theta^{\mathrm{b}} \mathrm{Ds}^{\mathrm{c}}$

$\mathrm{Na}$ equação (1), RP é a resistência à penetração (em $\mathrm{MPa}), \theta$ é o conteúdo de água do solo $\left(\mathrm{em}^{3} \mathrm{~cm}^{-3}\right)$, Ds é a densidade do solo (em $\mathrm{Mg} \mathrm{m}^{-3}$ ). Os coeficiente $\mathrm{a}, \mathrm{b}$ e c são os parâmetros do modelo obtidos mediante ajustes não-lineares, utilizando o método de Gauss-Newton (SAS Institute, 1991).

\section{RESULTADOS E DISCUSSÃO}

Os momentos estatísticos para as variáveis umidade $(\theta)$, densidade do solo (Ds) e resistência à penetração (RP) são apresentados na Tabela 1. A amplitude da variação da umidade volumétrica $(0,25$ a $0,47 \mathrm{~cm}^{3} \mathrm{~cm}^{-3}$ ), obtida neste estudo, concordaram com os valores extremos da curva de retenção ( $\psi-0,0005 \mathrm{MPa}$ e $\psi-1,5 \mathrm{MPa})$ obtidos no mesmo solo por Moraes et al. (1993). O parâmetro densidade do solo apresentou um coeficiente de variação próximo ao determinado por Silva et al. (1997), apesar de estes autores terem estudado a variação da densidade do solo na linha e na entrelinha da cultura de milho. Nielsen et al. (1973) constataram valores de coeficiente de variação de até $10 \%$ para a densidade do solo.

Em sistemas de pastejo intensivo, a probabilidade de que ocorra pisoteio repetidamente no mesmo local aumenta, promovendo acréscimos nos valores de densidade do solo que variam de $7 \%$ a $18 \%$ (Willatt \& Pullar, 1983; Chanasyk \& Naeth, 1995; Azenegashe et al., 1997). Comparando os resultados de densidade do solo deste estudo com os obtidos por Carvalho (1976), na mesma área, porém anteriormente à aplicação do sistema de pastejo rotacionado intensivo, observa-se que o incremento da densidade do solo foi de $12 \%$. A variabilidade desta propriedade do solo tende a ser mais elevada na camada superficial em virtude da influência dos fatores de manejo. A densidade do solo é influenciada principalmente 
pela idade e tipo de forrageira, pela taxa de lotação animal empregada, pela umidade do solo no momento do pastejo e pela textura do solo. O coeficiente de variação, neste estudo, pode estar associado ao efeito do pisoteio dos animais, que aumenta a densidade do solo, e ao efeito do sistema radicular das plantas, que promove uma redução dos valores dessa propriedade do solo. Um dos principais efeitos da atividade das raízes consiste na estruturação do solo, a qual implica uma diminuição da densidade do solo na rizosfera (Derner et al., 1997).

Os resultados da Tabela 1 demonstram que na área amostrada existe uma ampla variação dos dados de resistência à penetração, a qual pode ser atribuída ao gradiente de umidade imposto e variabilidade da densidade do solo. O procedimento utilizado para a obtenção da variação da umidade demonstrou ser adequado, visto que permitiu obter uma grande variação dos valores de resistência, necessária para o ajuste do modelo empregado.

A resistência do solo à penetração das raízes é drasticamente alterada quando ocorre degradação da estrutura do solo (Willatt \& Pular, 1983; Murphy et al., 1995), e constitui um indicador mais sensível do efeito do pisoteio dos animais na estrutura do que a densidade do solo (Chanasyk \& Naeth, 1995). Entretanto, os valores de resistência mecânica do solo variam em função da densidade e da umidade do solo, no momento em que foram efetuadas as medidas (Busscher et al., 1997). Interpretação mais correta dos dados de resistência à penetração pode ser feita pelo modelo proposto por Busscher (1990), já que esse modelo leva em consideração a influência da umidade e da densidade do solo na resistência mecânica.
A Tabela 2 mostra os parâmetros obtidos no ajuste da resistência à penetração em função da umidade e densidade do solo. O intervalo de confiança dos coeficientes b e c não incluem valor igual a zero, possibilitando afirmar que a resistência à penetração foi significativamente relacionada com ambas as variáveis, sendo negativamente influenciada pela umidade e positivamente pela densidade do solo (Glantz \& Slinker, 1990). Esse comportamento da resistência em relação a umidade e densidade é um processo bem descrito na literatura (Letey, 1985; Silva et al., 1994; Busscher et al., 1997) em diferentes tipos de solos e sistemas de manejo.

A representação gráfica dos dados de resistência ajustados é ilustrada na Fig. 1. A resistência à penetração foi estimada a partir da variação de umidade e densidade obtida nas amostras. Verifica-se que o secamento do solo em conjunto com a elevação da densidade provoca um aumento acentuado na resistência mecânica do solo. Valores críticos de resistência à penetração para o crescimento do sistema radicular de gramíneas tem sido reportados na literatura, e a maioria dos trabalhos concordam em assinalar um valor limite em torno de 2,5 MPa (Taylor \& Burnett, 1964; Semmel et al., 1990). Tal fato sugere que, na área estudada, a combinação de elevadas densidades com reduzida umidade do solo pode resultar na ocorrência de valores de resistência mecânica em níveis impeditivos para o crescimento do sistema radicular do capim-elefante.

Assumindo-se o valor de 2,5 MPa como o limite a partir do qual o crescimento das plantas é afetado, pode-se, a partir do modelo, fazer uma série de inferências a respeito da ocorrência de restrições para a produção do capim-elefante. O modelo ajustado

TABELA 1. Momentos estatísticos das variáveis umidade do solo, densidade do solo e resistência à penetração.

\begin{tabular}{lccccc}
\hline Variáveis $^{1}$ & Média & Desvio padrão & Mínimo & Máximo & $\mathrm{CV}(\%)$ \\
\hline$\theta\left(\mathrm{cm}^{3} \mathrm{~cm}^{-3}\right)$ & 0,39 & 0,04 & 0,25 & 0,47 & 11,39 \\
Ds $\left(\mathrm{g} \mathrm{cm}^{-3}\right)$ & 1,39 & 0,09 & 1,20 & 1,54 & 6,46 \\
$\mathrm{RP}(\mathrm{MPa})$ & 1,99 & 1,42 & 9,47 & 0,70 & 71,43 \\
\hline
\end{tabular}

1 : umidade volumétrica; Ds: densidade do solo; RP: resistência à penetração. 
foi utilizado para estimar a variação da resistência mecânica do solo para três valores de umidade: no potencial mátrico $(\Psi)$ de $-0,01 \mathrm{MPa}$, correspondente à capacidade de campo (Sanchez, 1981; Dexter, 1988); no $\Psi=-0,08 \mathrm{MPa}$, correspondente ao limite superior passível de ser medido com um tensiômetro no campo (Libardi, 1995) e no $\Psi=-0,3 \mathrm{MPa}$, correspondente a um estágio mais avançado de secamento do solo, contudo, inferior ao ponto de murcha permanente $(\Psi=-1,5 \mathrm{MPa})$. As estimativas da resistência mecânica, nessas condições de umidade e com a variação natural de densidade do solo, são apresentadas na Fig. 2

No $\Psi=-0,3 \mathrm{MPa}$, a resistência mecânica não atinge valores limitantes (maiores que 2,5 MPa), apesar

TABELA 2. Resultados do ajuste dos dados de resistência à penetração através do modelo proposto por Busscher (1990): $\mathbf{R P}=\mathbf{a} \theta^{\mathbf{b}} \mathbf{D} \mathbf{s}^{\mathbf{c}}$, sendo $\mathbf{R P}$ a resistência à penetração (MPa), $\theta$ a umidade volumétrica $\left(\mathrm{cm}^{3} \mathrm{~cm}^{-3}\right)$ e Ds a densidade do solo $\left(\mathrm{g} \mathrm{cm}^{-3}\right) \cdot \mathbf{R}^{2}=\mathbf{0 , 9 3}$.

\begin{tabular}{ccccc}
\hline Parâmetro & $\begin{array}{c}\text { Valor } \\
\text { estimado }\end{array}$ & $\begin{array}{c}\text { Erro } \\
\text { padrão }\end{array}$ & \multicolumn{2}{c}{$\begin{array}{c}\text { Intervalo de } \\
\text { confiança }\end{array}$} \\
\cline { 3 - 5 } & & & $\begin{array}{c}\text { Limite } \\
\text { inferior }\end{array}$ & $\begin{array}{c}\text { Limite } \\
\text { superior }\end{array}$ \\
\hline a & 0,030 & 0,004 & 0,021 & 0,039 \\
b & $-3,555$ & 0,104 & $-3,764$ & $-3,346$ \\
c & 2,154 & 0,377 & 1,394 & 2,915 \\
\hline
\end{tabular}

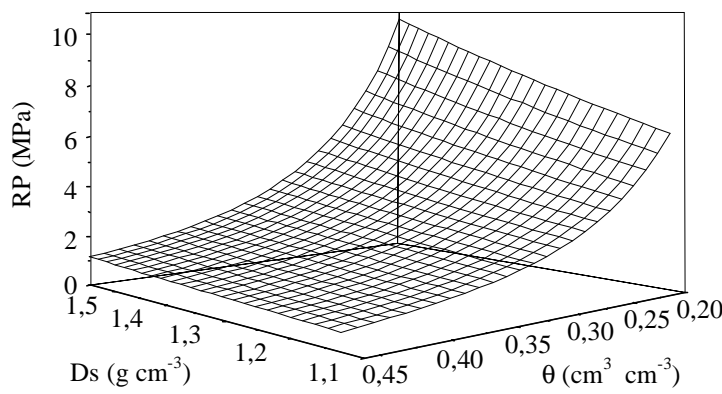

FIG. 1. Representação tridimensional dos valores de resistência à penetração $(R P)$ em função da umidade $(\theta)$ e densidade do solo (Ds). da variabilidade da densidade do solo (Fig. 2). Com o secamento do solo, a um nível de $\Psi=-0,08 \mathrm{MPa}$, ocorre aumento acentuado da resistência mecânica,
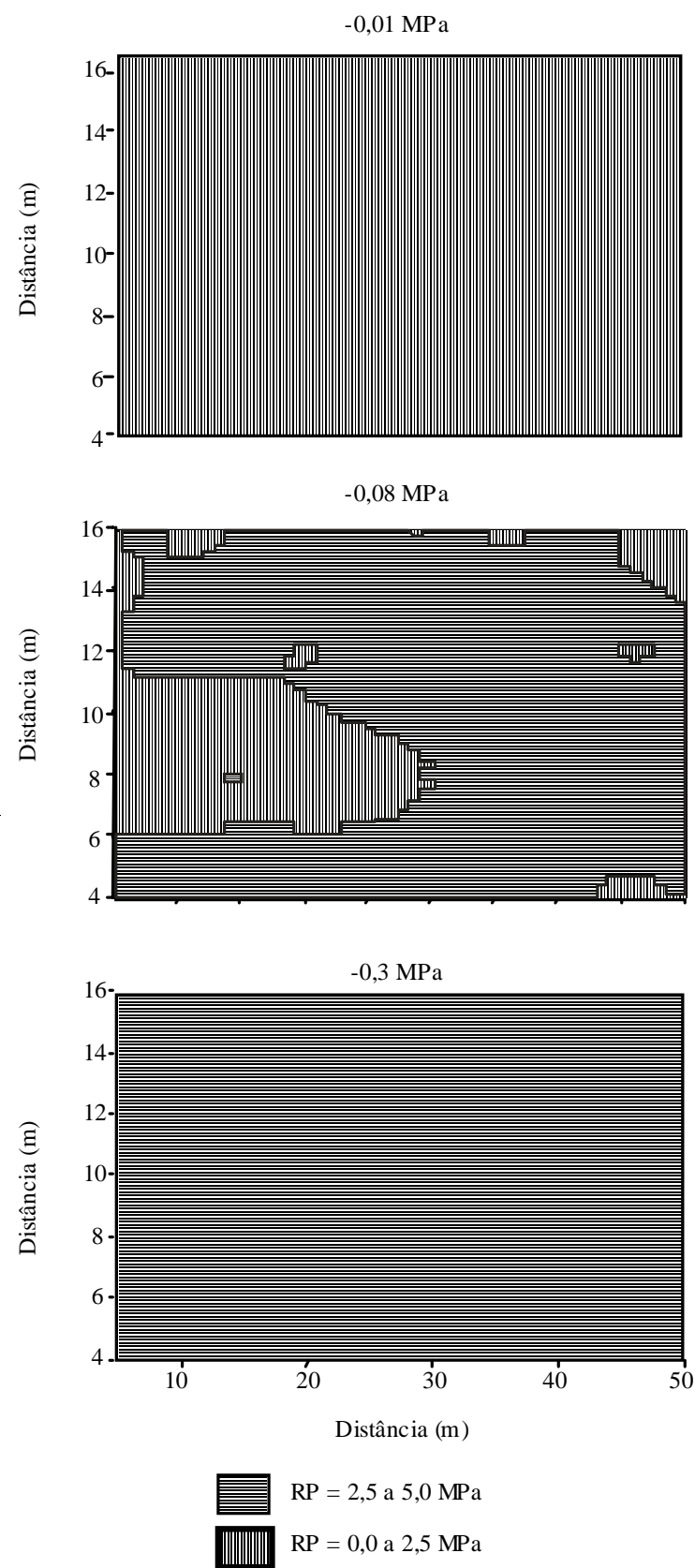

FIG. 2. Distribuição espacial da resistência à penetração (RP) nas umidades correspondentes aos potenciais de $-0,01 \mathrm{MPa},-0,08 \mathrm{MPa} e$ $-0,3$ MPa. 
com grande parte da área apresentando valores superiores a $2,5 \mathrm{MPa}$. As áreas com alta resistência mecânica correspondem às áreas com valores de densidade do solo mais elevadas. Valores de resistência do solo limitantes para o crescimento das raízes são atingidos em toda a área amostrada quando o $\Psi=-0,3 \mathrm{MPa}$. Esses resultados indicam que, na área estudada, a resistência mecânica do solo torna-se limitante sob potenciais entre $-0,08$ e $-0,3 \mathrm{MPa}$, entre os quais o conteúdo de água do solo provavelmente não é fator limitante ao crescimento das plantas. Entretanto, o controle da umidade pode ser utilizado como prática de manejo para o controle da resistência do solo, como foi demonstrado por Phene \& Beale (1972).

Os resultados sugerem que a umidade no solo deveria ser mantida acima de $0,349 \mathrm{~cm}^{3} \mathrm{~cm}^{-3}$ ( $\Psi=-0,08 \mathrm{MPa}$ ), para sustentar uma condição física do solo adequada ao crescimento das plantas. Valores de umidade próximos a $0,349 \mathrm{~cm}^{3} \mathrm{~cm}^{-3}$ podem refletir numa menor produção do capim-elefante, já que níveis de resistência mecânica maiores que $2,5 \mathrm{MPa}$ podem impor reduções na produção de biomassa do sistema radicular e da parte aérea das plantas (Davies \& Zhang, 1991; Tardieu, 1994). Isto implica que o $\Psi=-0,08 \mathrm{MPa}$, freqüentemente utilizado como limite crítico a partir do qual deve-se iniciar o fornecimento de água via irrigação, não é adequado para o sistema estudado, visto que nesse potencial a maior parte da área amostrada apresenta condições de resistência à penetração restritivas ao desenvolvimento radicular (Fig. 2).

Medidas adequadas da resistência mecânica do solo exigem o emprego de penetrômetros que possibilitem a obtenção de dados de resistência sob uma ampla variação de umidade, o que nem sempre é exequível no campo. Os penetrômetros de laboratório permitem a utilização de amostras indeformadas, nas quais podem ser controladas as variações da umidade sob a variação natural da densidade do solo, e possibilitam obter a curva de resistência do solo incorporando a variação natural da estrutura do solo.

A partir do conhecimento do modelo da curva de resistência do solo, podem ser estimadas as densidades do solo críticas $\left(\mathrm{D}_{\mathrm{sc}}\right)$ para o crescimento das plantas, definidas como aquelas em que a resistência à penetração atinge o valor crítico de $2,5 \mathrm{MPa}$.
Esses valores de densidades críticas são dependentes da umidade do solo, como indica o modelo ajustado. A relação densidade do solo crítica versus umidade volumétrica foi estimada a partir da equação 2, a qual foi resolvida utilizando-se um valor de resistência mecânica (RP) constante de 2,5 MPa e a variação de umidade obtida.

$D_{s c}=\left(R P / a \theta^{b}\right)^{1 / c}$.

Os resultados são apresentados na Fig. 3. Observa-se que a $D_{\mathrm{sc}}$ está positivamente relacionada com a umidade do solo. Quanto à umidade no $\Psi=-0,01 \mathrm{MPa}$, a $\mathrm{D}_{\mathrm{sc}}$ é de $1,70 \mathrm{~g} \mathrm{~cm}^{-3}$, ao passo que no $\Psi=-1,5 \mathrm{MPa}$ a $\mathrm{D}_{\mathrm{sc}}$ é de $1,05 \mathrm{~g} \mathrm{~cm}^{-3}$. Os resultados sugerem que, com o secamento do solo, ocorre um estreitamento da faixa de densidades em que a resistência é favorável ao crescimento das plantas (RP menor que 2,5 MPa).

A partir da Fig. 3, pode-se obter informações importantes para o manejo do solo sob o sistema de pastejo estudado. Conforme a umidade aumenta, podem ser admitidos valores de densidade do solo mais elevados, sem que o valor-limite de 2,5 MPa de resistência à penetração seja superado. A densidade do solo e o conteúdo de umidade do solo são parâmetros físicos que podem ser medidos rotineiramente, e a partir do conhecimento de qualquer um deles pode-se estabelecer o limite critico do outro. A qualidade física do solo adequada ao crescimento das plantas, em termos de resistência do solo à penetração das raízes, poderá ser obtida de duas for-

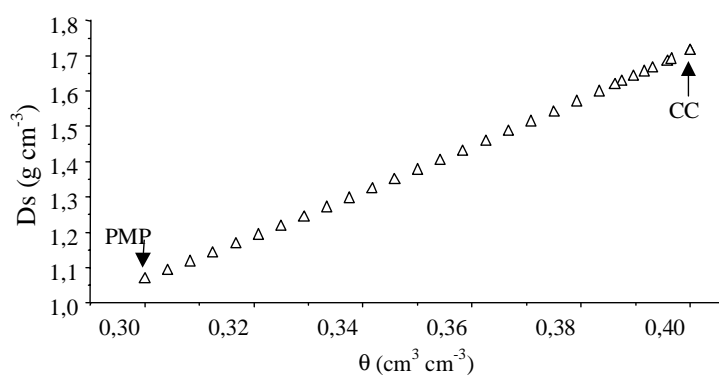

FIG. 3. Variação da densidade crítica do solo (Ds) em função da variação da umidade $(\theta)$ entre capacidade de campo e ponto de murcha permanente na resistência crítica de 2,5 MPa. 
mas: a) mediante práticas mecânicas para diminuir a densidade do solo, e b) pela prática de irrigação, mantendo a umidade do solo acima do nível critico determinado. Neste último caso, deve ser levado em consideração o nível de aeração requerido para o crescimento normal das plantas, visto que umidade e aeração são fatores do solo que estão inversamente relacionados. O valor de $10 \%$ de aeração foi assinalado como limite crítico inferior, a partir do qual as raízes começam a reduzir seu crescimento (Dexter, 1988; Tardieu, 1994). Os resultados indicam que para sistemas semelhantes ao estudado, constituídos de espécies perenes e que não possibilitam a realização de práticas mecânicas de controle da densidade do solo sem danificar as plantas, a melhoria e manutenção da qualidade física do solo para o crescimento das plantas depende do controle de umidade do solo. Este é um fator decisivo para atingir as máximas produtividades.

\section{CONCLUSÃo}

A curva de resistência do solo determinada é um parâmetro útil na avaliação da qualidade física do solo; permite identificar áreas com resistência mecânica potencialmente limitante ao crescimento das raízes e estabelecer a umidade e a densidade do solo críticas para o desenvolvimento das plantas.

\section{REFERÊNCIAS}

AZENEGASHE, O.A.; ALLEN, V.; FONTENOT, J. Grazing sheep and cattle together or separately: effect on soil and plants. Agronomy Journal, Madison, v.89, p.380-386, 1997.

BENGHOUGH, A.G.; MULLINS, C.E. Mechanical impedance to root growth: a review of experimental techniques and root growth responses. Journal of Soil Science, Oxford, v.41, p.341-358, 1990.

BLAKE, G.R.; HARTGE, K.H. Bulk density. In: KLUTE, A. (Ed.). Methods of soil analysis: physical and mineralogical methods. 2.ed. Madison : America Society of Agronomy, 1986. p.363-375.

BUSSCHER, W.J.Adjustment of flat-tipped penetrometer resistance data to a common water content. Transactions of the ASAE, Saint Joseph, v.3, n.2, p.519-524, 1990.
BUSSCHER, W.J.; BAUER, P.J.; CAMP, C.R.; SOJKA R.E. Correction of cone index for soil water content differences in a coastal plain soil. Soil and Tillage Research, Amsterdam, v.43, n.2, p.205-217, 1997.

CARVALHO, S.R. Influência de dois sistemas de manejo de pastagens na compactação de uma Terra Roxa Estruturada. Piracicaba : USP/ESALQ, 1976. 89p. Dissertação de Mestrado.

CASSEL, D.K.; BOWEN, H.D.; NELSON, L.A. An evaluation of mechanical impedance for three tillage treatments on Norfolk Loamy Sand. Soil Science Society of America. Journal, Madison, v.42, p.116120,1978 .

CHANASYK, D.S.; NAETH, M.A. Grazing impacts on bulk density and soil strength in the foothills fescue grasslands of Alberta, Canada. Canadian Journal of Soil Science, Ottawa, v.24, n.2, p.551-557, 1995.

DAVIES, W.J.; ZHANG, J. Root signals and the regulation of growth and development of plants in drying soil. Annual Review of Plant Physiology and Plant Molecular Biology, Palo Alto, v.42, p.55-76, 1991.

DERNER, J.D.; BRISKE, D.D.; BOUTTON, T.W. Does grazing mediate soil carbon and nitrogen accumulation beneath $\mathrm{C}_{4}$, perennial grasses along an environmental gradient? Plant and Soil, Dordrecht, v.191, n.2, p.147-156, 1997.

DEXTER, A.R. Advances in characterization of soil structure. Soil and Tillage Research, Amsterdam, v.11, n.1, p.199-238, 1988.

GLANTZ, S.A.; SLINKER, B.K. Primer of applied regression and analysis of variance. New York : McGraw-Hill, 1990. 777p.

GUPTA, S.C.; ALLMARAS, R.R. Models to assess the susceptibility of soils to excessive compaction. Advances in Soil Sciences, New York, v.6, p.65100, 1987.

HAAG, H.P.; DECHEN, A.R. Eficiências minerais em plantas forrageiras. In: PEIXOTO, A.M.; MOURA, J.C. de; FARIA, V.P. de (Ed.). Pastagens: fundamentos da exploração racional. Piracicaba : FEALQ, 1986. p.51-71.

HAKANSSON, I.; VOORHEES, W.B. Soil compaction. In: LAL, R.; BLUM, W.H.; VALENTINE, C.; STEWART, B.A. (Ed.). Methods for assessment of soil degradation. Boca Raton : CRC, 1998. p.167179.

Pesq. agropec. bras., Brasília, v.35, n.7, p.1493-1500, jul. 2000 
HAVEREN, B.B. van. Soil bulk density as influenced by grazing intensity and soil type on a shortgrass prairie site. Journal of Range Management, Denver, v.36, n. 5 , p.586-588, 1983

HORN, R.; LEBERT, M. Soil compactability and compressibility. In: SOANE, B.D.; OUWERKERK, C. van (Ed.). Soil compaction in crop production. Amsterdam : Elsevier, 1994. p.45-69. (Developments in Agricultural Engineering, 11).

HORTON, R.; ANKENY, M.D.; ALLMARAS, R.R. Effects of soil compaction on soil hydraulic properties. In: SOANE, B.D.; OUWERKERK, C. van (Ed.). Soil compaction in crop production Amsterdam : Elsevier, 1994. p.141-165. (Developments in Agricultural Engineering , 11)

KLUTE, A. Water retention: laboratory methods. In KLUTE, A. (Ed.). Methods of soil analysis: physical and mineralogical methods. Madison America Society of Agronomy, 1986. p.635-660

LETEY, J. Relationship between soil physical properties and crop production. Advances in Soil Sciences, New York, v.1, p.277-294, 1985

LIBARDI, P.L. Dinâmica da água no solo. Piracicaba : Libardi, 1995. 497p

MORAES, S.Q.; LIBARDI, P.L.; DOURADO NETO, D. Problemas metodológicos na obtenção da curva de retenção da água pelo solo. Scientia Agricola, Piracicaba, v.50, n.3, p.383-392, 1993.

MURPHY, W.M.; BARRETO, A.D.; SILMAN, J.P.; DINDAL, D.L. Cattle and sheep grazing effects on soil organisms, fertility and compaction in a smoothstalked meadow grass dominant white clover sward. Grass and Forrage Science, Oxford, v.50, p.191194, 1995

NIELSEN, D.R.; BIGGAR, J.W.; ERH, K.T. Spatial variability of field-measured soil water properties. Hilgardia, Berkeley, v.42, n.7, p.251-260, 1973.

OMETTO, J.C. Registros e estimativas de parâmetros meteorológicos da região de Piracicaba. Piracicaba : FEALQ, 1989. 76p.

PASSIOURA, J.B.; GARDNER, P.A. Control of leaf expansion in wheat seedlings growing in drying soil Australian Journal of Plant Physiology, Melbourne, v.17, p.149-157, 1990.

PHENE, C.J.; BEALE, O.W. High-frequency irrigation for water nutrient management in humid regions. Soil Science Society of America. Journal, Madison, v.40, p.430-436, 1972.

Pesq. agropec. bras., Brasília, v.35, n.7, p.1493-1500, jul. 2000
PROFFITT, A.P.B.; BENDOTTI, S.; HOWELL, M.R.; EASTHAM, J. The effect of sheep trampling and grazing on soil physical properties and pasture growth for a Red-Brown earth. Australian Journal of Agricultural Research, Melbourne, v.44, n.2, p.317-331, 1993.

SANCHEZ, P.A. Suelos del trópico: características y manejo. San Jose : IICA, 1981. 634p.

SAS INSTITUTE (Cary, Estados Unidos). Procedure guide for personal computers. 5.ed. Cary, 1991. $1104 \mathrm{p}$.

SEMMEL, H.; HORN, R.; HELL, U.; DEXTER, A.R.; SCHULZE, E.D. The dynamics of soil aggregate formation and the effect on soil physical properties. Soil Technology, Amsterdam, v.3, p.113-129, 1990

SILVA, A.P. da; KAY,B.D.; PERFECT, E. Characterization of the least limiting water range. Soil Science Society of America. Journal, Madison, v. 58 , p.1775-1781, 1994.

SILVA, A.P. da; KAY, B.D.; PERFECT, E. Management versus inherent soil properties effects on bulk density and relative compaction. Soil and Tillage Research, Amsterdam, v.44, n.1, p.81-93, 1997.

TARDIEU, F. Growth and functioning of roots and of root systems subjected to soil compaction: Towards a system with multiple signaling. Soil and Tillage Research, Amsterdam, v.30, n.2/4, p.217-243, 1994

TAYLOR, H.M.; BURNETT, E. Influence of soil strength on root growth habits of plants. Soil Science, Baltimore, v.98, p.174-180, 1964.

TORMENA, C.A.; SILVA, A.P. da; LIBARDI, P.L. Caracterização do intervalo hídrico ótimo de um Latossolo Roxo sob plantio direto. Revista Brasileira de Ciência do Solo, Campinas, v.22, n.4, p.573-581, 1998.

VEPRASKAS, M.J. Plant response mechanisms to soil compaction. In: WILKINSON, R. (Ed.). Plant environment interactions. New York : M. Dekker, 1994. p.263-287.

WARREN, S.D.; NEVILL, M.B.; BLACKBURN, W.H.; GARZA, N.E. Soil response to trampling under intensive rotation grazing. Soil Science Society of America. Journal, Madison, v.50, n.5, p.1336$1341,1986$.

WILLATT, S.T;; PULLAR, D.M. Changes in soil physical properties under grazed pastures. Australian Journal of Soil Research, Melbourne, v.22, n.4, p.343-348, 1983. 